\title{
Research on Capacitance Voltage Transformer Harmonic Measurement
}

\author{
Xin Shen, ${ }^{1 *}$ Hong-Chun Shu, ${ }^{1}$ and Min $\mathrm{Cao}^{2}$ \\ ${ }^{1}$ Mechanical and Electrical Engineering College, Kunming University of Science and Technology, \\ No. 727 Jingming South Road, Chenggong District, Kunming 650500, China \\ ${ }^{2}$ Yunnan Electric Power Research Institute, \\ No. 105, Yunda West Road, Guandu District, Kunming 650217, China
}

(Received April 20, 2018; accepted March 20, 2019)

Keywords: CVT, harmonic measurement, transmission characteristics, capacitive current method

According to the issue of transmission characteristics of a capacitor voltage transformer (CVT), which leads to the inaccuracy of power grid voltage harmonics values, a new test system and a method of measuring the capacitive current of a CVT were proposed. A comparison of the results of the conventional test method and actual power grid harmonics verified the validity and accuracy of the proposed test method, and a test of the voltage harmonics in the actual power grid showed that the test system can completely satisfy the requirements of testing the voltage harmonics.

\section{Introduction}

Currently, $110 \mathrm{kV}$ measurement systems are widely used to monitor the grid voltage on the secondary side of a transformer. Owing to disadvantages such as the ferromagnetic resonance with line distributed capacitance, large size, and high cost, electromagnetic voltage transformers (VTs) cannot meet the needs of a power system. Compared with electromagnetic VTs, a capacitor voltage transformer (CVT) has a large electric field strength margin and a reliable insulation. A voltage-dividing capacitor also serves as a coupling capacitor in a high-frequency carrier channel and does not form a ferromagnetic resonance with the switch port capacitance. In addition, the advantages of small size, light weight, less maintenance work, and low cost result in the wide use of the CVT. In foreign countries, CVTs are mostly used at voltages above $72.5 \mathrm{kV}$. In China, CVTs are widely used at $110 \mathrm{kV}$ and above. ${ }^{(1)}$

Although CVTs meet the requirements of the system used in the fundamental voltage measurement, system protection, and fundamental signal conversion of automatic devices, owing to the characteristics of CVT transmission, CVTs cannot correctly reflect the actual situation in the system with harmonic components. In measuring the voltage harmonics of the system, the secondary side voltage signal obtained by a CVT transformation device has a large error and the harmonic measurement data cannot be adopted. Therefore, the national standard GB/T 14549-1993 "grid harmonic" appendix D $7^{(2)}$ states that "a CVT cannot be used for harmonic measurement," and IEC standards and other related technical reports are also *Corresponding author: e-mail: 23755803@qq.com https://doi.org/10.18494/SAM.2019.2270 
provided. Therefore, how to test and grasp accurate grid harmonic levels and exact voltage distortion data through CVTs, what measurement method to adopt, and what test equipment to use for harmonic voltage testing have become the focus of power quality supervision of the power grids, and this is the primary problem that should necessarily be solved. ${ }^{(3)}$

\section{Analysis of the Harmonic Characteristics of CVT}

The CVT composed of voltage dividing capacitors, compensation reactors, intermediate transformers, dampers, and loads is shown in Figs. 1 and $2 .{ }^{(4-6)}$ In essence, it is mainly a capacitive voltage divider; in Fig. $1, \mathrm{C}_{1}$ is a high-voltage capacitor and $\mathrm{C}_{2}$ is a medium-voltage capacitor. The partial pressure ratio of the CVT is

$$
n=\frac{u_{1}}{u_{2}}=\frac{C_{1}+C_{2}}{C_{1}}+\frac{1}{\omega^{2} C_{1} L} .
$$

Compared with the partial pressure of the capacitive divider, the partial pressure ratio of the CVT is higher by $\Delta n$, that is, $\Delta n=1 /\left(\omega^{2} C_{1} L\right)$, as shown in Eq. (1). $\Delta n$ is equal to the ratio of the capacitive reactance of the CVT low-voltage arm to the inductive reactance of the TV and is related to the frequency, i.e., when the frequency is different, the ratio of $\Delta n$ is also different. The TV equivalent inductor $L$ is a nonlinear element whose size varies with the operating voltage and frequency. Therefore, the CVT variable ratio differs at different frequencies. From Eq. (1), the amplitude- and phase-frequency characteristics of the transfer functions of a multiple $220 \mathrm{kV}$ CVT are obtained and shown in Fig. 3.

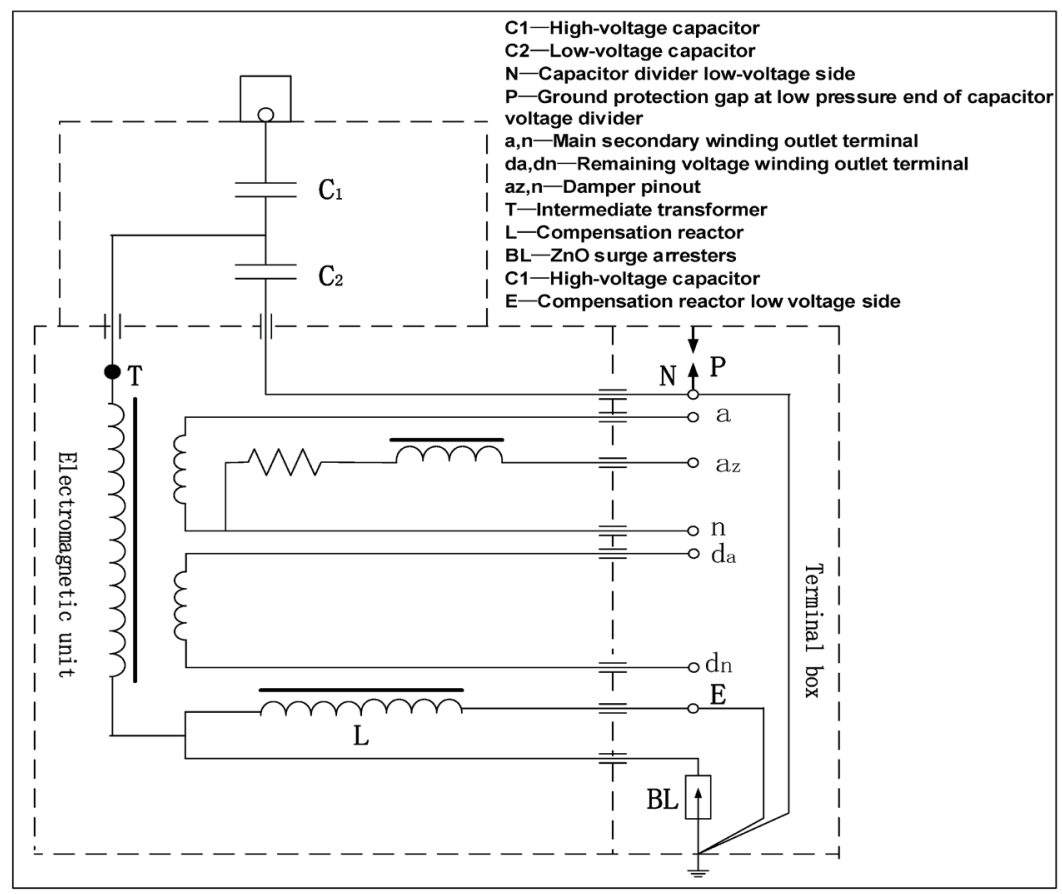

Fig. 1. Structure of CVT. 


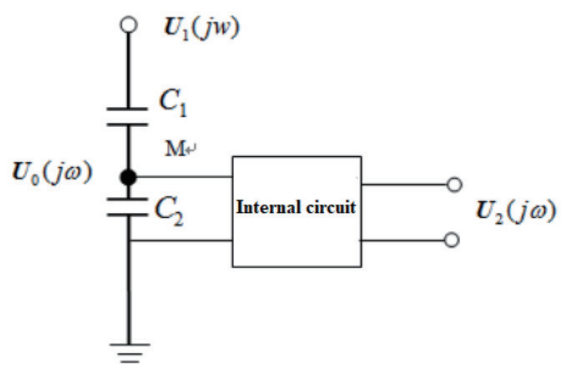

Fig. 2. Equivalent circuit diagram.

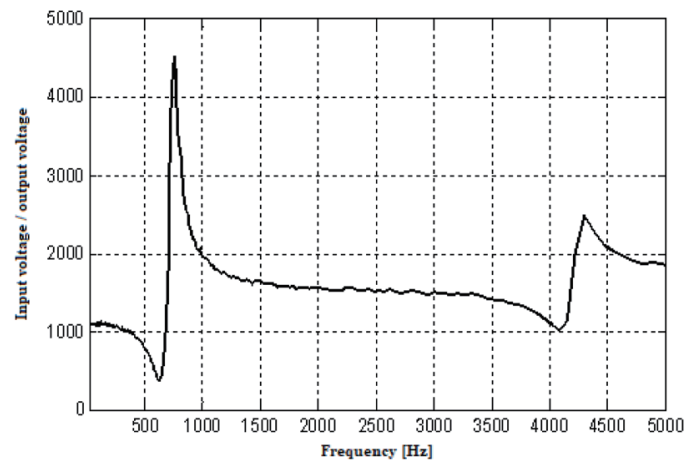

(a)

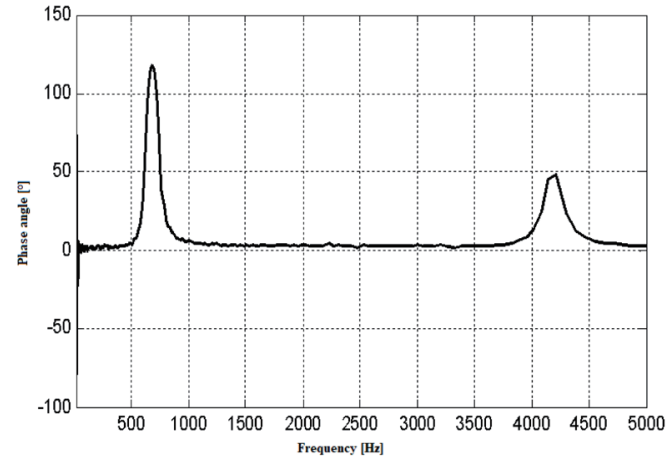

(b)

Fig. 3. (a) Amplitude- and (b) phase-frequency characteristics of transfer functions of multiple $220 \mathrm{kV} \mathrm{CVT.}$

According to the amplitude- and phase-frequency characteristics of the CVT, it can be seen that the CVT has different transformation ratios at different frequencies. Therefore, both lowand high-order harmonics have a certain filtering effect, which is essentially a band-pass filter system, and for different CVTs, owing to the different components of the parameter values, the initial and cut-off frequencies are different. Therefore, it is inevitable that distortion occurs in the harmonic measurement when the voltage signal is collected from the secondary side of the CVT. $^{(7-9)}$

\section{CVT Harmonic Measurement Based on Capacitive Current}

At present, the method of measuring grid voltage harmonics based on the CVT is to obtain the voltage signal from the secondary side of the CVT and combine the harmonic measurement and analysis instrument for harmonic analysis. ${ }^{(10-12)}$ To prevent the distortion of the harmonic components caused by the CVT transmission characteristics, some scholars have proposed a method of obtaining the CVT transfer function and then correcting the harmonic measurement offline. Commonly used methods of obtaining the CVT transfer function are the network analyzer method, high-voltage harmonic injection method, and the like. Owing to the different transmission characteristics of different CVTs, this method should test the transfer function of each CVT, which is theoretically feasible, but the project is actually not suitable for generalization. 
To solve the technical problem that a CVT cannot be used in harmonic measurement, a method of calculating the grid voltage by measuring high- and medium-voltage capacitive currents flowing through a CVT is proposed according to the principle of a CVT and the analysis of the characteristics of capacitive reactance that changes with frequency. The feasibility and limitation of this method are verified. Finally, a CVT harmonic measurement system based on the capacitive current method is designed.

\subsection{Measuring principle}

The method of testing CVT harmonics based on capacitive current follows Kirchhoff's voltage principle, a high-precision current sensor is loaded on the CVT capacitor branch to test the high- and medium-voltage capacitive currents flowing through the CVT, ${ }^{(13-16)}$ and the grid voltage is calculated by combining the CVT high- and medium-voltage capacitive reactances for further analysis. The equivalent circuit is shown in Fig. 4.

In Fig. 3, the high-precision current sensors $\mathrm{A}$ and $\mathrm{B}$ respectively measure the currents flowing in the high-voltage capacitor $\mathrm{C}_{1}$ and medium-voltage capacitor $\mathrm{C}_{2}$, and the voltage $U_{1}(j \omega)$ on the primary side is calculated as

$$
U_{1}(j \omega)=I_{1}(j \omega) j X_{c 1}+I_{2}(j \omega) j X_{c 2} .
$$

Here, $U_{1}(j \omega)$ is the primary-side voltage; $I_{1}(j \omega)$ is the total current flowing through the highand medium-voltage capacitors and the internal circuit at the frequency point, which can be measured by the current coil B; $I_{c 2}$ is the in-ground current flowing through the medium-voltage capacitor $\mathrm{C}_{2}$ at the frequency point, which can be measured by the current coil $\mathrm{A}$. $X_{c 1}(j \omega)$ is the

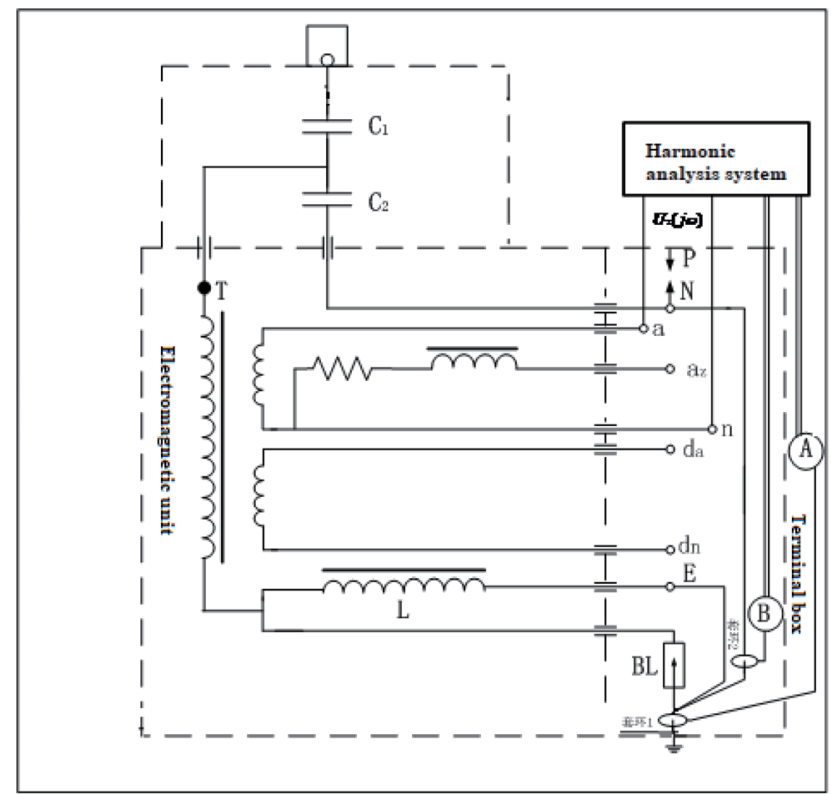

Fig. 4. Equivalent circuit diagram of capacitive current method. 
reactance of the high-voltage capacitor $\mathrm{C}_{1}$ at the frequency point, and $X_{c 2}(j \omega)$ is the reactance of the medium-voltage capacitor $\mathrm{C}_{2}$ at this frequency point.

\subsection{Test of CVT high- and medium-voltage capacitors $C_{1}$ and $C_{2}$}

The realization of the CVT harmonic testing method based on capacitive current requires that the capacitive reactance has a linear relationship with the reciprocal of the frequency in the concerned harmonics test frequency band (up to $5 \mathrm{kHz}$ ).

To verify this requirement, the impedance of the $110 \mathrm{kV} \mathrm{CVT} \mathrm{(TYD1} 10 / \sqrt{3}-0.02 \mathrm{H}$ ) used for testing was measured using an impedance analyzer, Agilent 4294A (working frequency: 40-110 $\mathrm{MHz}$ and resolution: $1 \mathrm{mHz}$ ).

The test method is described as follows. During the test, disconnect the connection line between the electromagnetic unit and the midpoint of the capacitor, and connect the impedance analyzer to the CVT high-voltage capacitor $\mathrm{C}_{1}$ and middle-voltage capacitor $\mathrm{C}_{2}$. As shown in Fig. 5 , the capacitances of the test high-voltage capacitor $\mathrm{C}_{1}$ and medium-voltage capacitor $\mathrm{C}_{2}$ vary with frequency.

Impedance parameters of TYD1 $10 / \sqrt{3}-0.02 \mathrm{H}$ were tested using Agilent 4294A. The test frequency range is $40-5 \mathrm{kHz}$.

The impedance test results of the high- and medium-voltage capacitors $C_{1}$ and $C_{2}$ are shown in Fig. 6. By using these test results, the changes in $C_{1}$ and $C_{2}$ capacitances with the frequency are obtained and shown in Fig. 7.

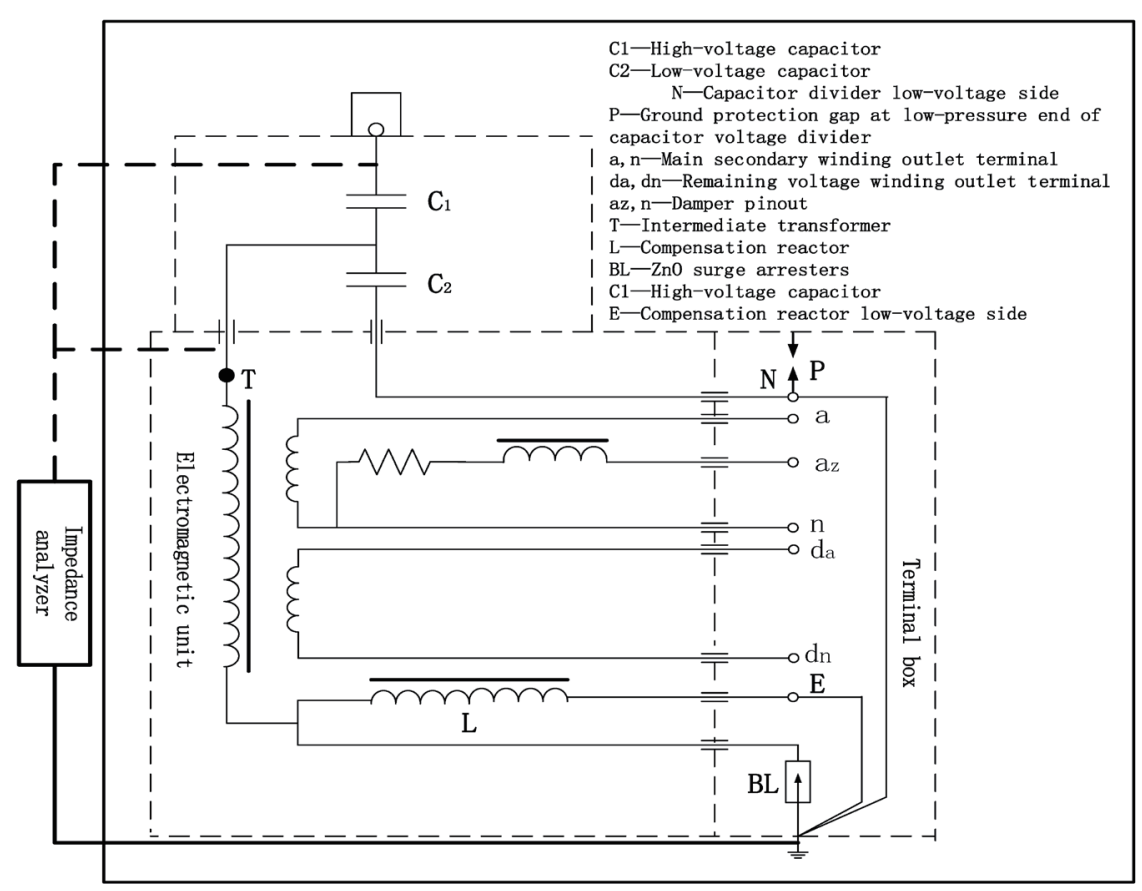

Fig. 5. Principle diagram of the CVT capacitive reactance measurement. 


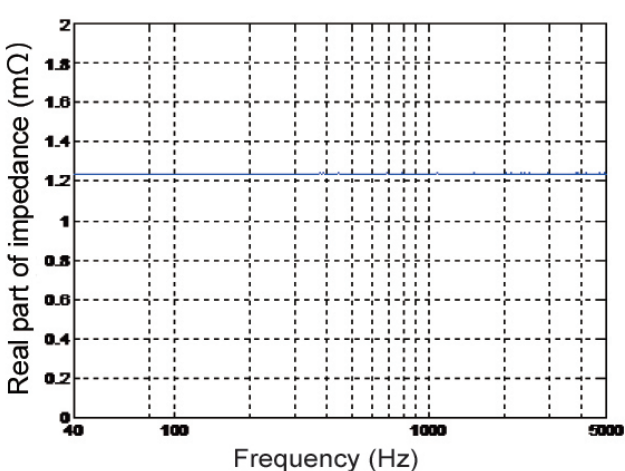

(a)

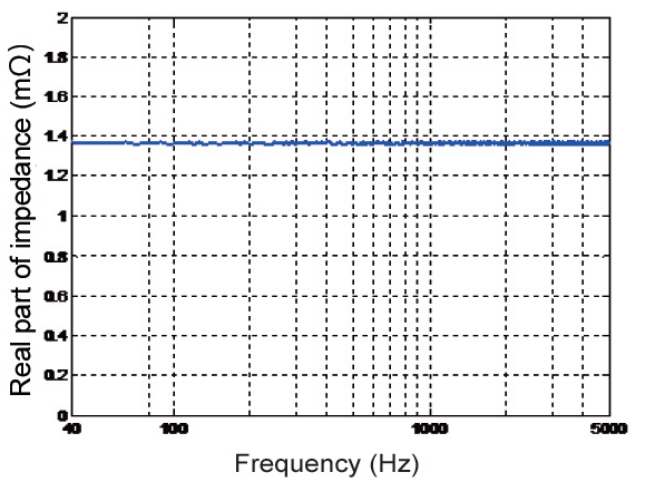

(c)

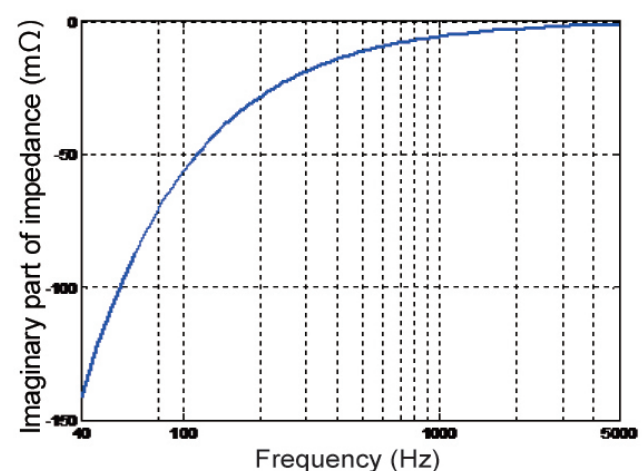

(b)

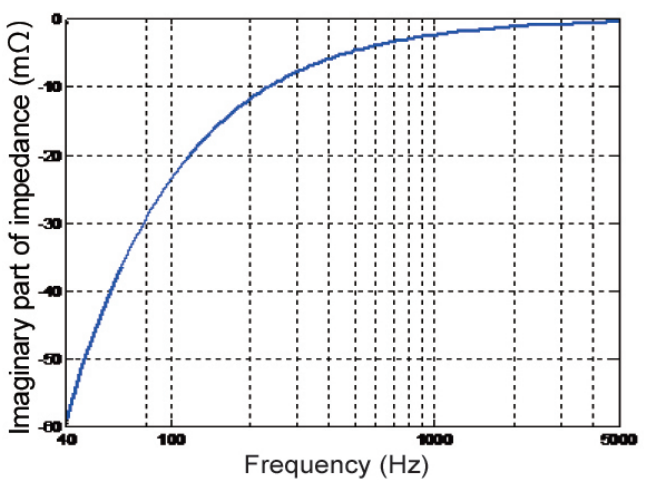

(d)

Fig. 6. (Color online) Test results of CVT high- and medium-voltage capacitor $\mathrm{C}_{1}$ and $\mathrm{C}_{2}$ impedances.

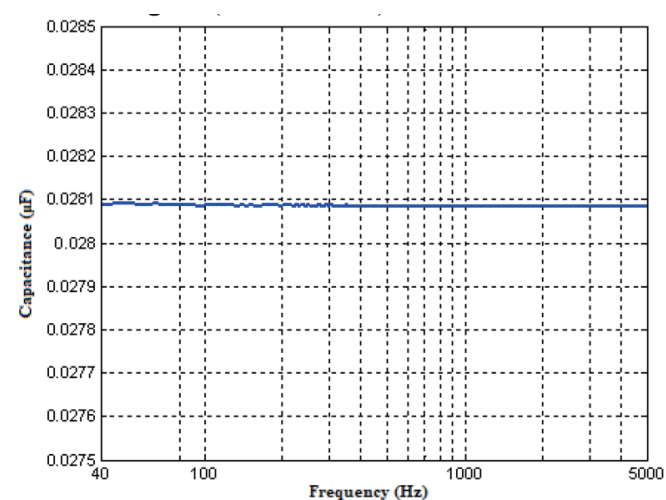

(a)

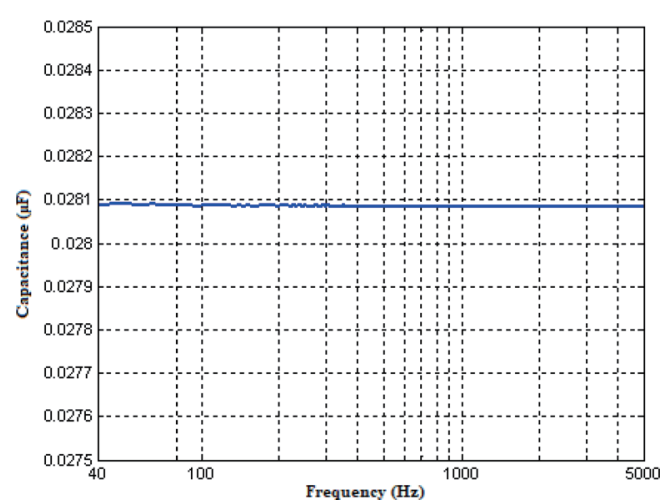

(b)

Fig. 7. (Color online) Changes in frequency capacitances of CVT high- and medium-voltage capacitosr $\mathrm{C}_{1}$ and $\mathrm{C}_{2}$ : (a) high-pressure side of $\mathrm{C}_{1}$ and (b) high-pressure side of $\mathrm{C}_{2}$.

The test results show that the capacitance of the CVT high-voltage capacitor $\mathrm{C}_{1}$ is 0.028 $087 \mu \mathrm{F}$, which is in accordance with the value of the given parameter $\mathrm{C}_{1}(0.028095 \mu \mathrm{F})$. The capacitance of the CVT medium voltage capacitor $\mathrm{C}_{2}$ is $0.067485 \mu \mathrm{F}$, which is consistent with the value of the given parameter $\mathrm{C}_{2}(0.06755 \mu \mathrm{F})$. 
Note that there is a resistive component in the measurement due to the existence of the lead during the test, but the maximum resistance does not exceed $1.4 \mathrm{~m} \Omega$, and the test result is not affected compared with the capacitive reactance exceeding $1 \mathrm{k} \Omega$ at $5 \mathrm{kHz}$. In the frequency band below $5 \mathrm{kHz}$, the capacitance is not affected by stray parameters, lead resistance and inductance, but only the capacitance characteristics. Therefore, the currents flowing through CVT high- and medium-voltage capacitors are tested; moreover, it is feasible to measure the grid voltage by calculating the capacitive reactances of CVT high- and medium-voltage capacitors.

\subsection{Design of CVT harmonic testing system based on capacitive current}

According to the test principle of CVT harmonics based on the capacitive current method, the harmonic test system designed in this study is shown in Fig. 8. Because the currents of CVT high- and medium-voltage capacitors are at the microlevel, the current sensor used is an Agilent N2820A probe. The Agilent N2820A probe offers a high sensitivity that is 200 times higher than the sensitivity of a clamp-on current probe that can only measure sub-milliamps current. The probe bandwidth ranges from DC to $500 \mathrm{kHz}$ and the minimum measurable current is 250 $\mu \mathrm{A}$, which will be wound on the wire under test for many turns to amplify the measured current in the test, simultaneously reducing the space circuit area, to prevent external interference. A data acquisition card is used for each channel $250 \mathrm{kHz}$ sampling rate, 12-bit resolution, with the simultaneous sampling of the 4-channel data acquisition card.

The CVT equivalent circuit is simplified, and the secondary load is equivalent to the primary side, the simplified equivalent circuit shown in Fig. 9. Here, $U_{1}$ is the primary-side line voltage, $U_{2}$ is the primary voltage of the electromagnetic unit, $\mathrm{C}_{1}$ and $\mathrm{C}_{2}$ are partial pressure capacitors, $L$ is the compensation reactor, $L_{M}$ is the intermediate transformer excitation reactance, and $R_{x}$ is the equivalent load impedance. According to the simplified equivalent circuit, $U_{1}$ is calculated from $U_{2}$ and the current $I_{L}$ of the compensation reactor $L$ is obtained as

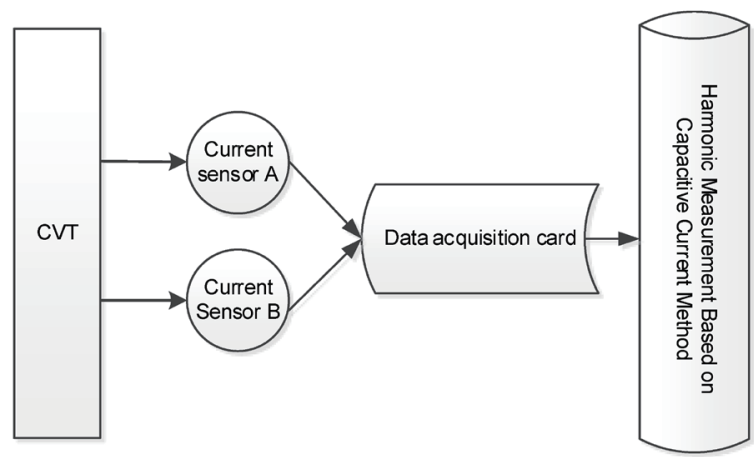

Fig. 8. Structure diagram of CVT harmonic test system based on capacitive current method.

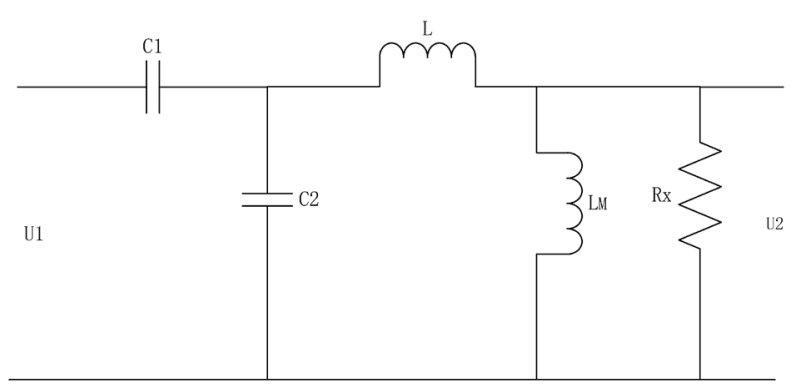

Fig. 9. CVT simplified equivalent circuit diagram. 


$$
I_{L}=\frac{U_{2}}{R x}+\frac{U_{2}}{2 \pi f L_{M} j} .
$$

The voltage $U_{c 2}$ of the capacitor $\mathrm{C}_{2}$ is

$$
U_{c 2}=U_{2}+\left(\frac{U_{2}}{R x}+\frac{U_{2}}{2 \pi f L_{M} j}\right) \times 2 \pi f L j .
$$

The current $I_{c 2}$ of the capacitor $\mathrm{C}_{2}$ is

$$
I_{c 2}=\frac{U_{c 2}}{-\frac{1}{2 \pi f C_{2}} j}=\frac{U_{2}+\left(\frac{U_{2}}{R x}+\frac{U_{2}}{2 \pi f L_{M} j}\right) \times 2 \pi f L j}{-\frac{1}{2 \pi f C_{2}} j} .
$$

The current $I_{c 1}$ of the capacitor $\mathrm{C}_{1}$ is

$$
I_{c 1}=I_{c 2}+I_{L}=\frac{U_{2}+\left(\frac{U_{2}}{R x}+\frac{U_{2}}{2 \pi f L_{M} j}\right) \times 2 \pi f L j}{-\frac{1}{2 \pi f C_{2}} j}+\frac{U_{2}}{R x}+\frac{U_{2}}{2 \pi f L_{M} j} .
$$

The primary-side voltage $U_{1}$ is

$$
\begin{aligned}
U_{1}=U_{c 1}+U_{c 2}= & \left(\frac{U_{2}+\left(\frac{U_{2}}{R x}+\frac{U_{2}}{2 \pi f L_{M} j}\right) \times 2 \pi f L j}{-\frac{1}{2 \pi f C_{2}} j}+\frac{U_{2}}{R x}+\frac{U_{2}}{2 \pi f L_{M} j}\right) \\
& \times\left(-\frac{1}{2 \pi f C_{1}} j\right)+U_{2}+\left(\frac{U_{2}}{R x}+\frac{U_{2}}{2 \pi f L_{M} j}\right) \times 2 \pi f L j .
\end{aligned}
$$

Assuming that the transformation ratio of the electromagnetic unit is the $P_{\text {electromagnetic unit }}$, the overall transformation ratio of the CVT is 


$$
\begin{aligned}
P_{C V T}= & P_{\text {electromagnetic unit }} \times \frac{U_{1}}{U_{2}} \\
= & P_{\text {electromagnetic unit }} \times\left\{\left(\frac{1+\left(\frac{1}{R x}+\frac{1}{2 \pi f L_{M} j}\right) \times 2 \pi f L j}{-\frac{1}{2 \pi f C_{2}} j}+\frac{1}{R x}+\frac{1}{2 \pi f L_{M} j}\right)\right. \\
& \left.\times\left(-\frac{1}{2 \pi f C_{1}} j\right)+1+\left(\frac{1}{R x}+\frac{1}{2 \pi f L_{M} j}\right) \times 2 \pi f L j\right\} .
\end{aligned}
$$

By using this equation, the variation characteristics of the overall transformation ratio $P_{C V T}$ of the CVT with the frequency are experimentally studied under saturation and nonsaturation conditions.

\section{Experiment and Test}

Several 110 and $220 \mathrm{kV}$ CVTs were tested with the CVT harmonic current measurement system based on capacitive current. The principle of the test is shown in Fig. 10. ${ }^{(17-22)}$

The test method is described as follows. The high-voltage harmonic source is used to inject the harmonic with a ratio of $10 \%$ into the CVT. The order of the harmonic is 2-50. Owing to

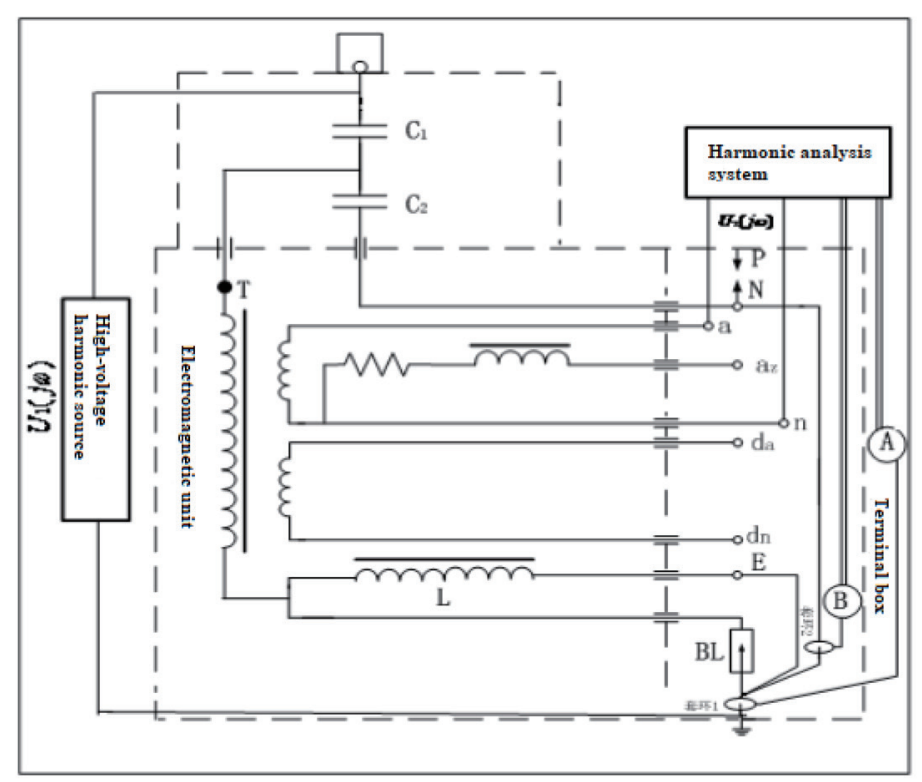

Fig. 10. Testing connection diagram. 
space limitation, the article lists only one $220 \mathrm{kV} \mathrm{CVT}$ test result. The test result of the CVT through the high- and medium-voltage capacitor currents is shown in Fig. 11.

The CVT primary voltage after injection and the CVT primary voltage calculated using Eq. (2) are shown in Fig. 12.

The frequency spectrum of the voltage shown in Fig. 12 is analyzed, and the obtained results are shown in Fig. 13.

It can be concluded that, when the percentage of primary harmonic voltage is $10 \%$, the calculated percentage of primary voltage harmonic is close to $10 \%$ with a small error. Table 1 gives the numerical results of the harmonic test. The harmonic percentage measured by the capacitive current method is close to the harmonic level of the primary side of the CVT injected at the time of testing. The maximum deviation of the harmonic percentage is $1.89 \%$.

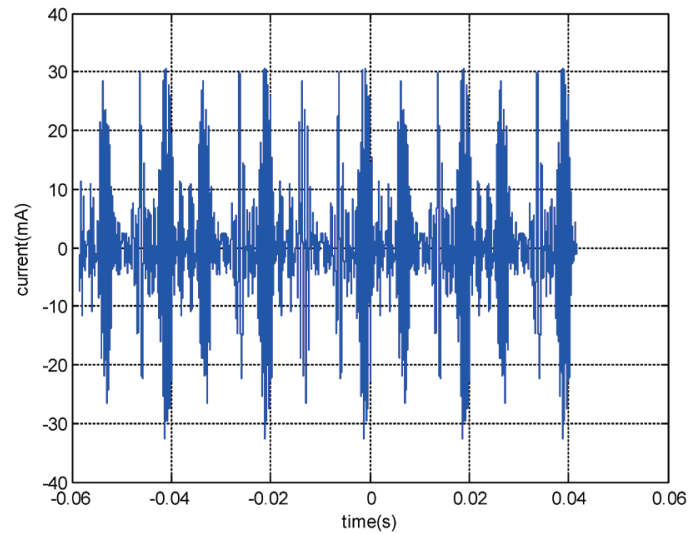

(a)

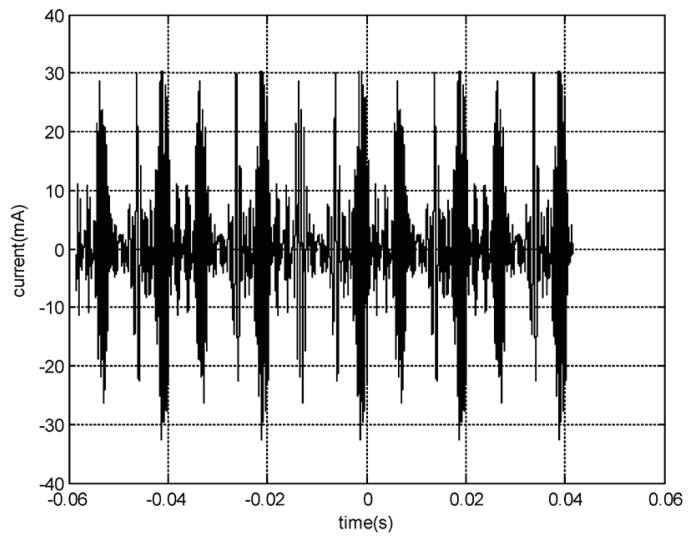

(b)

Fig. 11. (Color online) Curve diagram of current flowing through (a) high- and (b) medium-voltage capacitors.

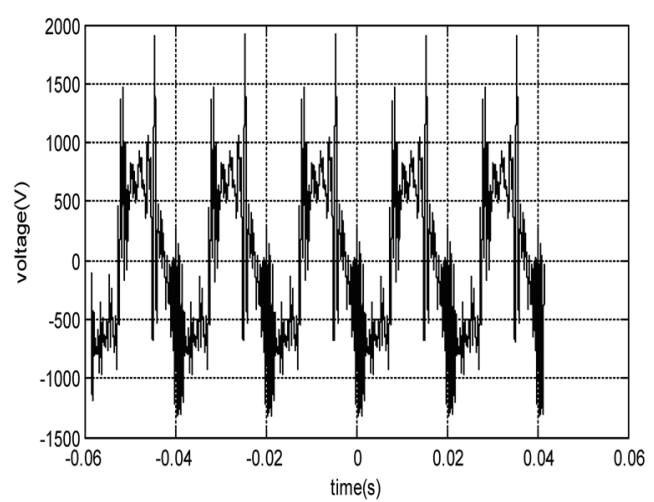

(a)

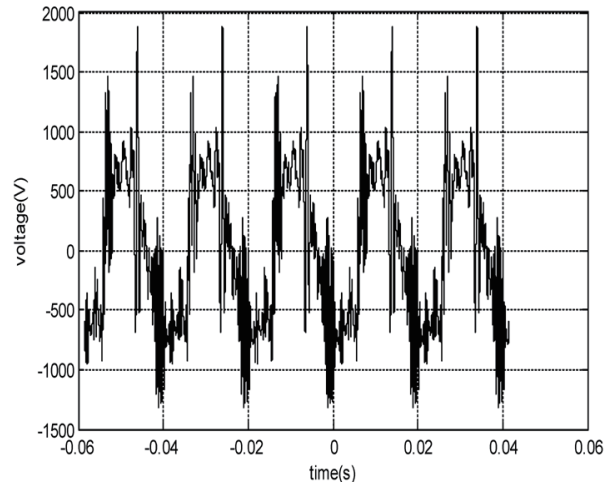

(b)

Fig. 12. Comparison of CVT high voltages (time domain). (a) CVT primary voltage after injection. (b) CVT primary voltage calculated using Eq. (2). 


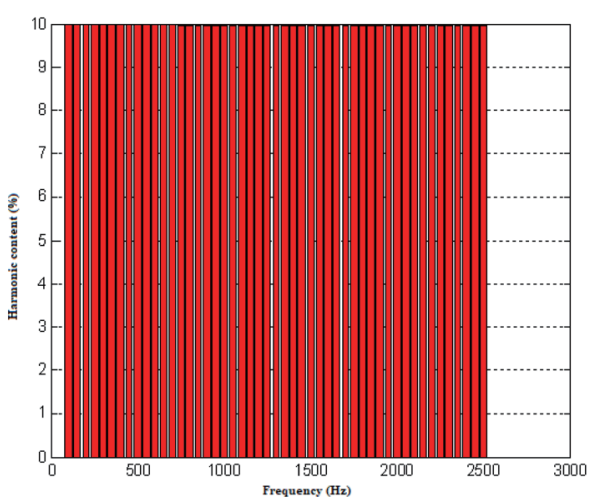

(a)

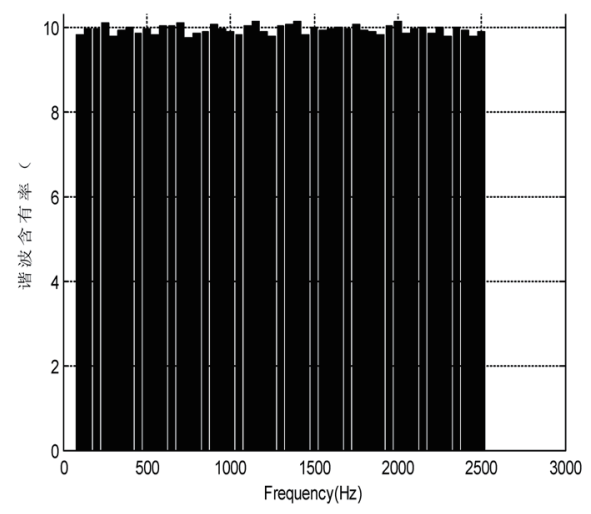

(b)

Fig. 13. (Color online) Comparison of CVT high voltages (frequency domain). (a) CVT primary voltage after injection. (b) CVT primary voltage calculated using Eq. (2).

Table 1

Harmonic errors.

\begin{tabular}{|c|c|c|c|c|c|c|c|}
\hline $\begin{array}{l}\text { Harmonic } \\
\text { (order) }\end{array}$ & $U_{l}(j w)(\mathrm{V})$ & $U_{2}(j w)(\mathrm{V})$ & $\delta(\%)$ & $\begin{array}{l}\text { Harmonic } \\
\text { (order) }\end{array}$ & $U_{l}(j w)(\mathrm{V})$ & $U_{2}(j w)(\mathrm{V})$ & $\delta(\%)$ \\
\hline 1 & 220.00 & 220.00 & - & 27 & 22.07 & 22.21 & 0.62 \\
\hline 3 & 21.99 & 22.41 & 1.89 & 29 & 21.99 & 22.13 & 0.63 \\
\hline 5 & 21.99 & 22.24 & 1.12 & 31 & 22.02 & 22.33 & 1.43 \\
\hline 7 & 21.99 & 22.21 & 0.99 & 33 & 22.03 & 22.08 & 0.24 \\
\hline 9 & 21.98 & 22.30 & 1.46 & 35 & 22.00 & 22.07 & 0.34 \\
\hline 11 & 21.98 & 22.01 & 0.12 & 37 & 22.05 & 22.37 & 1.43 \\
\hline 13 & 21.96 & 22.18 & 0.98 & 39 & 22.04 & 22.21 & 0.77 \\
\hline 15 & 22.03 & 22.37 & 1.56 & 41 & 22.00 & 22.32 & 1.44 \\
\hline 17 & 22.03 & 22.20 & 0.76 & 43 & 22.01 & 22.03 & 0.08 \\
\hline 19 & 22.02 & 22.22 & 0.91 & 45 & 21.93 & 22.05 & 0.56 \\
\hline 21 & 22.02 & 22.04 & 0.1 & 47 & 21.97 & 22.12 & 0.67 \\
\hline 23 & 22.02 & 22.31 & 1.31 & 49 & 21.93 & 22.05 & 0.54 \\
\hline 25 & 22.01 & 22.04 & 0.13 & & & & \\
\hline
\end{tabular}

Note: $U_{1}(j w)$ is the primary-side injection voltage of the CVT during the test, $U_{2}(j w)$ is the calculated CVT primaryside voltage, and $\delta$ is the relative deviation of the harmonic ratio.

\subsection{Comparison with traditional harmonic testing methods}

To further analyze the validity of the CVT harmonic detection method based on capacitive current, the harmonic test of the above $220 \mathrm{kV}$ CVT is carried out by using the conventional test method, that is, the same high-voltage harmonic is injected into the primary side of the CVT as the CVT harmonic testing method based on capacitive current. The CVT secondary voltage signal is derived for harmonic analysis. Finally, the test results of the two methods are compared. The results of such a comparison are shown in Figs. 14 and 15.

From Figs. 14 and 15, it can be seen that the results obtained by the traditional test methods have larger deviations than those obtained by the method of injecting the voltage with a harmonic content of $10 \%$ into the primary side of the CVT during the test. However, the results 


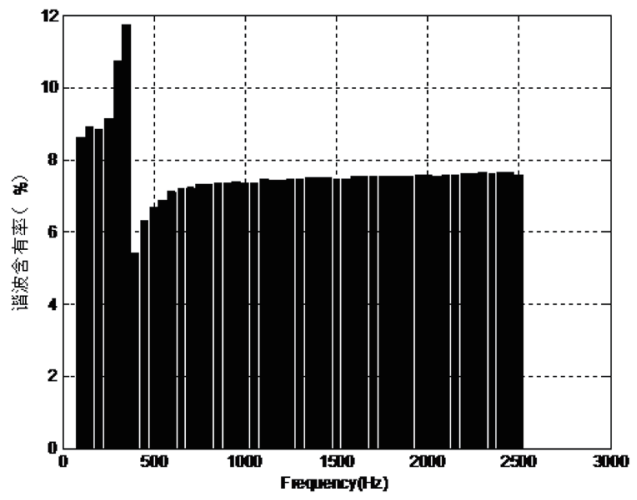

(a)

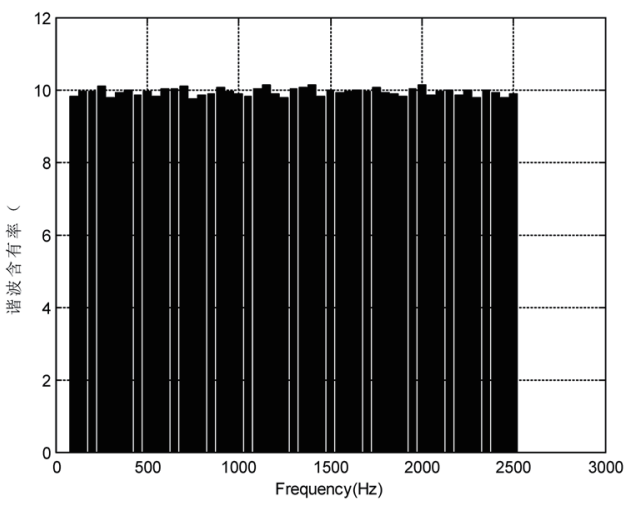

(b)

Fig. 14. Comparison of CVT high voltages (frequency domain). (a) Test results of traditional test method. (b) Capacitive current test results.

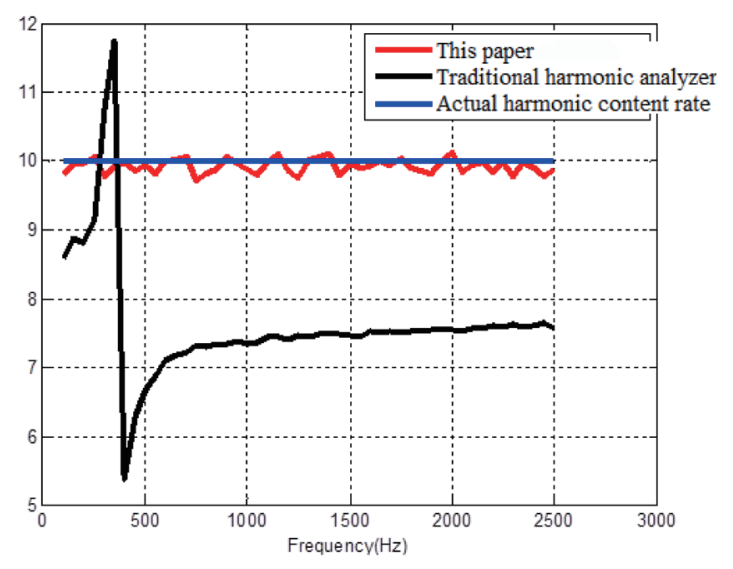

Fig. 15. (Color online) Comparison of conventional harmonic test data.
Table 2

Comparison of test results of two methods.

\begin{tabular}{lccccc}
\hline Harmonic (order) & 1 & 3 & 5 & 7 & 9 \\
\hline $\begin{array}{c}\text { Error of traditional } \\
\text { method (\%) }\end{array}$ & - & 23.45 & 35.98 & 24.58 & 36.70 \\
$\begin{array}{c}\text { Error of capacitive } \\
\text { current method (\%) }\end{array}$ & - & 1.89 & 1.12 & 0.99 & 1.46 \\
\hline Harmonic (order) & 11 & 13 & 15 & 17 & 19 \\
\hline $\begin{array}{c}\text { Error of traditional } \\
\text { method (\%) }\end{array}$ & 21.23 & 21.56 & 27.67 & 18.34 & 16.21 \\
$\begin{array}{c}\text { Error of capacitive } \\
\text { current method (\%) }\end{array}$ & 0.12 & 0.98 & 1.56 & 0.76 & 0.91 \\
\hline Harmonic (order) & 21 & 23 & 25 & 27 & 29 \\
\hline $\begin{array}{c}\text { Error of traditional } \\
\text { method (\%) }\end{array}$ & 2.94 & 3.41 & 1.92 & 3.15 & 2.29 \\
$\begin{array}{c}\text { Error of capacitive } \\
\text { current method (\%) }\end{array}$ & 0.10 & 1.31 & 0.13 & 0.62 & 0.63 \\
\hline Harmonic (order) & 31 & 33 & 35 & 37 & 39 \\
\hline $\begin{array}{c}\text { Error of traditional } \\
\text { method (\%) }\end{array}$ & 12.56 & 19.67 & 18.33 & 11.25 & 17.68 \\
$\begin{array}{c}\text { Error of capacitive } \\
\text { current method (\%) }\end{array}$ & 1.43 & 0.24 & 0.34 & 1.43 & 0.77 \\
\hline Harmonic (order) & 41 & 43 & 45 & 47 & 49 \\
\hline $\begin{array}{c}\text { Error of traditional } \\
\text { method (\%) }\end{array}$ & 17.32 & 13.13 & 16.43 & 17.85 & 15.53 \\
$\begin{array}{c}\text { Error of capacitive } \\
\text { current method (\%) }\end{array}$ & 1.44 & 0.08 & 0.56 & 0.67 & 0.54 \\
\hline & & & & & \\
\hline
\end{tabular}

of the CVT harmonic test based on capacitive current have a very small deviation from those obtained by the method of injecting the harmonic into the primary side of the CVT during the test. Table 2 shows the numerical results of the comparison of the two test methods. The maximum deviation of the harmonic content obtained by the traditional method is $35.98 \%$. The harmonic content measured based on the capacitive current method is close to the harmonic level of the primary side of the CVT injected during the test, with a maximum deviation of $1.89 \%$. 


\section{Conclusions}

We focus on solving the problem that a traditional high-voltage system voltage harmonic measurement method does not consider the wide-band characteristics of a CVT and leads to inaccurate measurement results. On the basis of the principle of a CVT and the characteristic of capacitive reactance that changes with frequency, a capacitive current method for accurately measuring the voltage harmonics of the power grid by a CVT is proposed. By comparing the test results of the traditional harmonic test method and the actual harmonics of the power grid, the correctness and accuracy of the capacitive current method are verified. Finally, a test system based on the capacitive current method to accurately test the voltage harmonics of the power grid is designed. The harmonic test method based on capacitive current is safe and reliable, shows an anti-interference ability, accurately measures the harmonic level of the power system by a CVT, and eliminates the distortion and drift caused by a CVT in harmonic measurements, providing reliable data for harmonic control. The maximum deviation of the harmonic content obtained by the traditional method is $35.98 \%$. The harmonic content based on the capacitive current method is close to that of the primary side of the CVT injected under test, with a maximum deviation of $1.89 \%$. The method can be applied to the random testing and on-line monitoring of the harmonics of EHV, HV, and MV grids. The testing method is simple, reliable, and safe, and has solved the problem that a CVT cannot be applied to harmonic measurements, as specified in the GB/T14549-1993 "Power Quality Utility Harmonic".

\section{References}

1 X. Ni, Y. Yan and H. Lin: High Voltage Eng. 32 (2006) 104.

2 Standard Voltages, Current Ratings and Frequencies. GB/T 14549—1993 Quality of electric energy supply harmonics in public supply network (Standards Press of China, Beijing, 1994).

3 M. Xu, H. Gao, and G. Zou: Autom. Electric Power Syst. 35 (2011) 71.

4 M. H. Zare, A. Mirzaei, and H. A. Abyaneh: 20th Iranian Conf. Electr. Eng. (ICEE, 2012) 523-528.

5 Q. Ma, X. Cui, and C. Ren: High Voltage Eng. 40 (2014) 1828.

6 Y. Wen and S. Chen: Power Capacitor React. Power Compensation 35 (2014) 45.

7 H. Wang, X. Wang, and X. Chen: Electr. Meas. Instrum. 47 (2010) 39.

8 D. Wang and J. Wang: High Voltage Eng. 27 (2001) 66.

9 T. Ding, Z. Chen, and J. Zhang: Electr. Meas. Instrum. 46 (2009) 9.

10 F. Ghassemi and PhilipGale: IEEE Trans. Power Delivery 20 (2005) 443.

11 L. Li, X. Duan, and G. Liu: Hebei Electr. Power 23 (2014) 52.

12 G. Liu and L. Li: Heilongjiang Electr. Power 36 (2014) 100.

13 W. Si, L. He, and H. Jin: Adv. Technol. Elect. Eng. Energy 32 (2013) 43.

14 M. Kezunovic, C. W. Fromen, and S. L. Nilsson: IEEE Trans. Power Delivery 7 (1992) 1927.

15 L. Kojovicl, M. Kezunovic, and C. W. Fromen: IEEE Trans. Power Delivery 9 (1994) 1907.

16 H. Gao, Q. Li, and X. Yu: Power Syst. Technol. 33 (2013) 3125.

17 R. Wang: Research on Distortion Measurement Using CVT (North China Electric Power University, Baoding, 2008).

18 Y. Zhang: Research on Transient Overreach of Capacitor Voltage Transformer (Zhejiang University, Hangzhou, 2005).

19 L. Wang and B. Fang: High Voltage Eng. 38 (2012) 2389.

20 S. Wu, X. Wang, and X. Li: High Voltage Eng. 35 (2009) 973.

21 X. Hw: Modern Power Quality Measurement Technology (Fluke Corporation, Beijing, 2012) 208.

22 C. Zhou: Power Capacitor 2 (2005) 43.

23 E. Mohns: 2012 Conf. Precision Electromagnetic Measurements (CPEM) (IEEE, 2012) 304-305. 


\section{About the Authors}

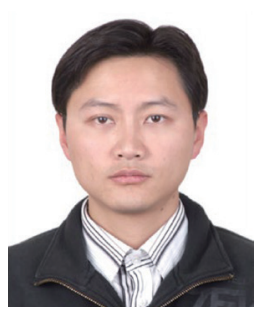

Xin Shen received his B.S. and M.S. degrees from Kunming University of Science and Technology, China, in 2003 and 2012 respectively. From 2003 to 2009, he was an assistant professor at Yunnan Electric Power Research Institute, China. Since 2017, he has been a professor at Yunnan Electric Power Research Institute. His research interests are in sensors, electric energy metering, and engineering.

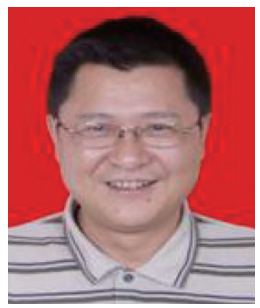

Hongchun Shu received his Ph.D. degree from the Harbin Institute of Technology, China, in 1997. From 1997 to 1998, he was an assistant professor at Kunming University of Science and Technology, China. Since 1998, he has been a professor at Kunming University of Science and Technology, China. His research interests are in power system automation, sensors, HVDC and flexible dc transmission, and distribution network fault detection and protection.

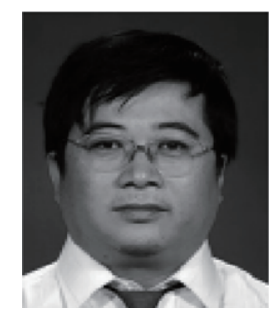

Min Cao received his B.S. degree from Kunming University of Science and Technology, China, in 1982. From 1988 to 2012, he was an assistant professor at Yunnan Electric Power Research Institute, China. Since 2012, he has been a professor at Yunnan Electric Power Research Institute, China. His research interests are in electric energy metering, engineering, and sensors. 